\title{
Pediatric Asthma Situation in Chengdu, China, During the COVID-19 Pandemic: An Observational Study
}

\author{
Lei Zhang \\ Xinglu Wang \\ Yijie Huang \\ Tao Ai \\ Huiling Liao \\ Jie $\mathrm{Hu}$ \\ Wei Tang \\ Yuanyuan Huang
}

Department of Pediatric Pulmonology, Chengdu Women's and Children's Central Hospital, School of Medicine, University of Electronic Science and Technology of China, Chengdu, People's Republic of China
Correspondence: Lei Zhang; Tao Ai Chengdu Women's and Children's Central Hospital, School of Medicine, University of Electronic Science and Technology of China, Chengdu, People's Republic of China

Email534167313@qq.com;

Aitaol108@163.com
Background: To tackle the COVID-19 pandemic and mitigate viral transmission, mainland China has implemented various disease prevention measures and arrangements. We hypothesize that these measures may be pose challenges to the management of pediatric asthma. Here, we studied the situation of pediatric asthma in Chengdu during the COVID-19 pandemic and compared the pediatric asthma situation before so as to provide a reference for designing effective management plan for pediatric asthma patients in the future facing the outbreak of epidemic.

Methods: An observational study was done to compare the pediatric asthma situation in Chengdu from 2017 to 2019 to the situation under COVID-19 pandemic. Asthma incidence, severe asthma attack, air quality, temperature, and patient follow-up were examined.

Results: The number of monthly asthma cases decreased significantly in February 2020. The number of asthma cases in 2017-2019 positively correlated with levels of particulate matter $\mathrm{PM}_{2.5}(\mathrm{p}=0.006)$ and $\mathrm{PM}_{10}(\mathrm{p}=0.005)$, while it negatively correlated with temperature $(\mathrm{p}=$ 0.048 ). No correlation was identified in 2020. A higher rate of severe asthma attack cases $(9.69 \%)$ was observed among asthma patients in $2020(\mathrm{p}=0.014)$. Differences were identified between the monthly severe asthma attack during the period 2017-2020 $(p<0.001)$. The rate of severe asthma attack cases peaked in June and September 2020 . The percentage of patients who failed to undergo pulmonary function tests was $34.5 \%$ in 2020 , remarkably higher than in the previous 3 years $(\mathrm{p}<0.001)$.

Conclusion: The situation and management of pediatric asthma during the 2020 COVID-19 pandemic differed from those in previous years, with more emphasis placed on disease prevention practices and facilities. To design future pediatric asthma management practice, the environmental and psychological impact on asthma management should also be considered. Local areas should make good use of telemedicine to manage pediatric asthma effectively.

Keywords: asthma, pediatric, COVID-19

\section{Background}

In November 2019, COVID-19 was first reported in Wuhan, China. Since then, its causative agent SARS-CoV-2 has spread everywhere in the world. The rapid deterioration of infected patients, their high mortality rate and the high communal transmission rate have quickly turned this infectious disease into a pandemic and public health emergency.

Many disease prevention measures have been implemented in China with an aim to mitigate transmission. During the COVID-19 pandemic, strict COVID-19-related disease prevention policies have been implemented in Chengdu, China (Table 1). 
Table I COVID-19-Related Disease Prevention Measures and Policies in Chengdu, China

\begin{tabular}{|c|c|}
\hline Date & Prevention Measure \\
\hline $24 / 1 / 2020$ & $\begin{array}{c}\text { Sichuan Province activated Urgent Handling of Public } \\
\text { Health Emergency mechanism }\end{array}$ \\
\hline $25 / 1 / 2020$ & $\begin{array}{l}\text { The Education Department of Sichuan Province } \\
\text { announces the suspension of all off-campus } \\
\text { training until effective control of COVID-19 pandemic }\end{array}$ \\
\hline $6 / 2 / 2020$ & $\begin{array}{l}\text { Announcement of the Guidelines on Disease } \\
\text { Prevention for Kindergartens as well as Primary, } \\
\text { Secondary and } \\
\text { Higher Education in Sichuan Province }\end{array}$ \\
\hline $6 / 2 / 2020$ & $\begin{array}{c}\text { Suspension of all education institutions in Sichuan } \\
\text { Province until late February }\end{array}$ \\
\hline $8 / 4 / 2020$ & $\begin{array}{l}\text { The Department of Education in Sichuan Province } \\
\text { announces that the date for resuming schools depends } \\
\text { on the progress of disease prevention. } \\
\text { Sufficient personal hygiene products should be available } \\
\text { at schools and restaurants }\end{array}$ \\
\hline $6 / 5 / 2020$ & Resumption of different kinds of education institutions \\
\hline $2 / 9 / 2020$ & $\begin{array}{c}\text { Memo about the disease prevention measures for } \\
\text { COVID-19 pandemics in autumn and winter } \\
\text { from the Department of Civil Affairs of Sichuan } \\
\text { Province }\end{array}$ \\
\hline $9 / 12 / 2020$ & $\begin{array}{c}\text { The Commission of Health announces that there are } \\
\text { three medium-risk areas in Sichuan Province. } \\
\text { Relevant personnel should not leave the area, in } \\
\text { principle. }\end{array}$ \\
\hline
\end{tabular}

While the disease prevention measures have effectively dampened disease transmission in Chengdu, this has severely challenged conventional management of chronic diseases, such as pediatric asthma. Populations will undeniably have to fight with viruses for a long while, in addition to social and environmental problems. In view of the profound impacts of COVID-19 pandemic brought to the world, better preventive measures should be formulated in order to protect vulnerable pediatric asthma patients.

In the present study, we performed an observational study to compare the pediatric asthma situation before and during the COVID-19 pandemic in order to provide a reference for effectively managing pediatric asthma in the future.

\section{Method and Materials}

This study was conducted within Chengdu Women's and Children's Hospital, Chengdu, China. The data of the patients suffering from asthma who had been confirmed and followed up were collected from 2017 to 2020. The diagnosis of asthma and severe asthma attacks was based on Guidelines for Bronchial Asthma Prevent and Management (2020 edition) ${ }^{1}$ and the Recommendations for Diagnosis and Management of Bronchial Asthma in Children (2020). ${ }^{2}$ A "big data" database source and extraction was constructed through the merging of multiple individual-level databases, included the Hospital information system (HIS), Laboratory Information System (LIS), Electronic Medical Record (ERM) and Nursing Information System (NIS) of Chengdu Women's and Children's Central Hospital, which was a special code identifier and the language exchange program using extract-transform loading (ETL) to standardize and effectively combine the data from multiple sources. The data about $\mathrm{PM}_{2.5}$ (particulate matter in the air $<2.5 \mathrm{um}$ ), $\mathrm{PM}_{10}$ (particulate matter in the air $<10 \mu \mathrm{m}$ ) and temperature were provided by the Chengdu Meteorological Service. The data of asthma incidence, severe asthma attack, air quality, temperature, and patient follow-up were analyzed. The study was approved by the ethics committee of Chengdu Women's and Children's Central Hospital Ethics Number B2021(5).

SPSS 25.0 software was employed for the statistical analysis in this study. Continuous, normally distributed data were reported as mean $\bar{x}$ ) \pm standard deviation (s). Skewed data were reported as median and interquartile range. Categorical data were reported in quantities and percentages. Pearson's chi-square test was used to assess differences in categorical data. The Pearson correlation analysis was used to study the correlation between asthma and $\mathrm{PM}_{2.5}, \mathrm{PM}_{10}$ and temperature. Associations were quantified as odds ratios (ORs) and $95 \%$ confidence intervals (CIs). All tests were two-tailed, and $\mathrm{p}<0.05$ was considered statistically significant.

\section{Results}

Information on pediatric asthma patients aged 3-18 years was collected. A total of 26,482 cases were collected from the system, comprising 16,250 males and 10,232 females, from January 2017 to December 2020. The numbers of asthma cases among children aged 3-18 years were 5608, 6784,7420 and 6670, respectively. The pattern of monthly pediatric asthma cases in 2020 was found to be different from the patterns in 2017-2019, with a significant decrease in February 2020 (Figure 1). 


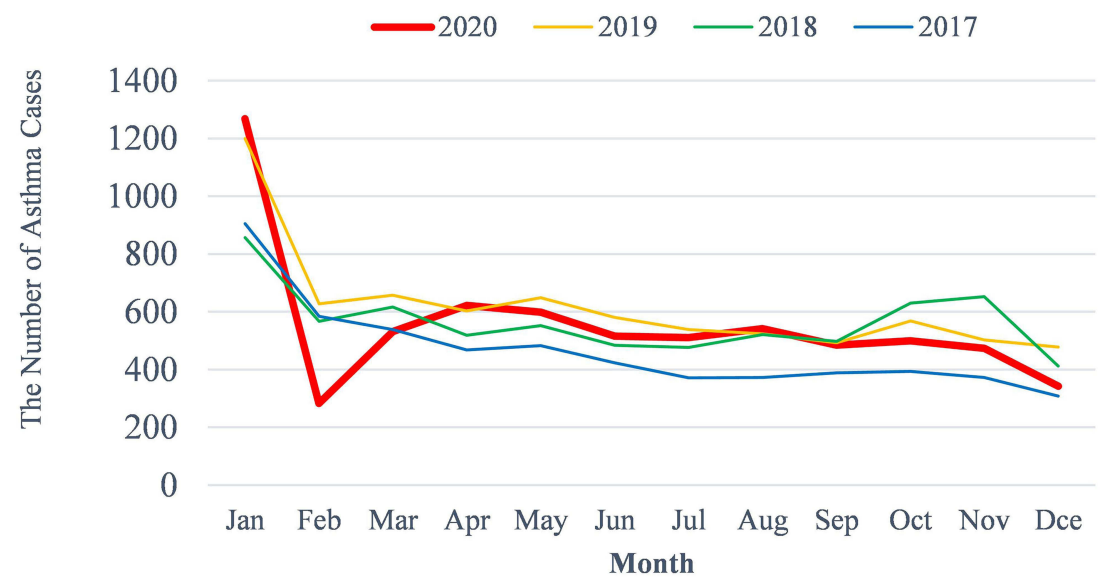

Figure I The number of monthly pediatric asthma patients.

From 2017 to 2019, the number of asthma cases was found to be positively correlated with levels of particulate matter PM $2.5(R=0.446, p=0.006)$ (Figure 2) and PM10 $(R=0.456, p=0.005)$ (Figure 3$)$, while it negatively correlated with temperature $(R=-0.322, p=$ 0.048) (Figure 4, Figure 5, Figure 6,Figure 7). The number of asthma cases in 2020 was found to have no correlation with PM2.5, PM10 or temperature (Figures 5-7).
The rate of severe asthma attack in 2020 was 9.69\%, higher than in 2019 (8.07\%), 2018 (5.85\%) and $2017(4.78 \%)$. Significant differences in the incidence of severe asthma attack were observed across the 4 years $(F=11.052 \mathrm{P}=0.014$ ) (Figure 8 ). Differences were identified between the monthly severe asthma attack incidents during the period 2017-2020: 2017 $(F=40.4288, \quad \mathrm{p}<0.001), 2018 \quad(F=74.2423$, $\mathrm{p}<0.001), 2019(F=34.0665, \mathrm{p}=0.004)$, and 2020

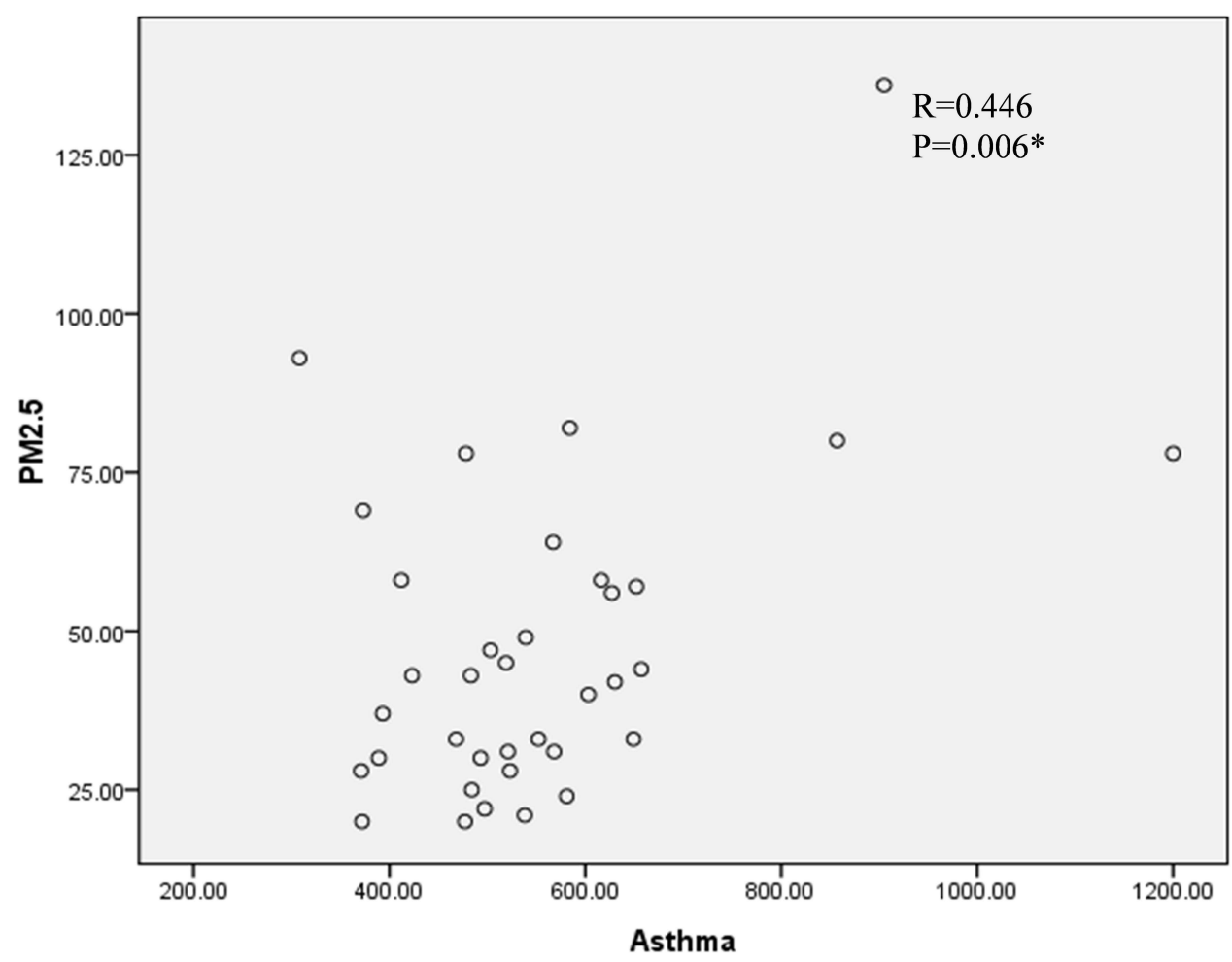

Figure 2 Correlation between the number of pediatric asthma patients and PM2.5 from 2017 to 2019 (*p<0.05). 


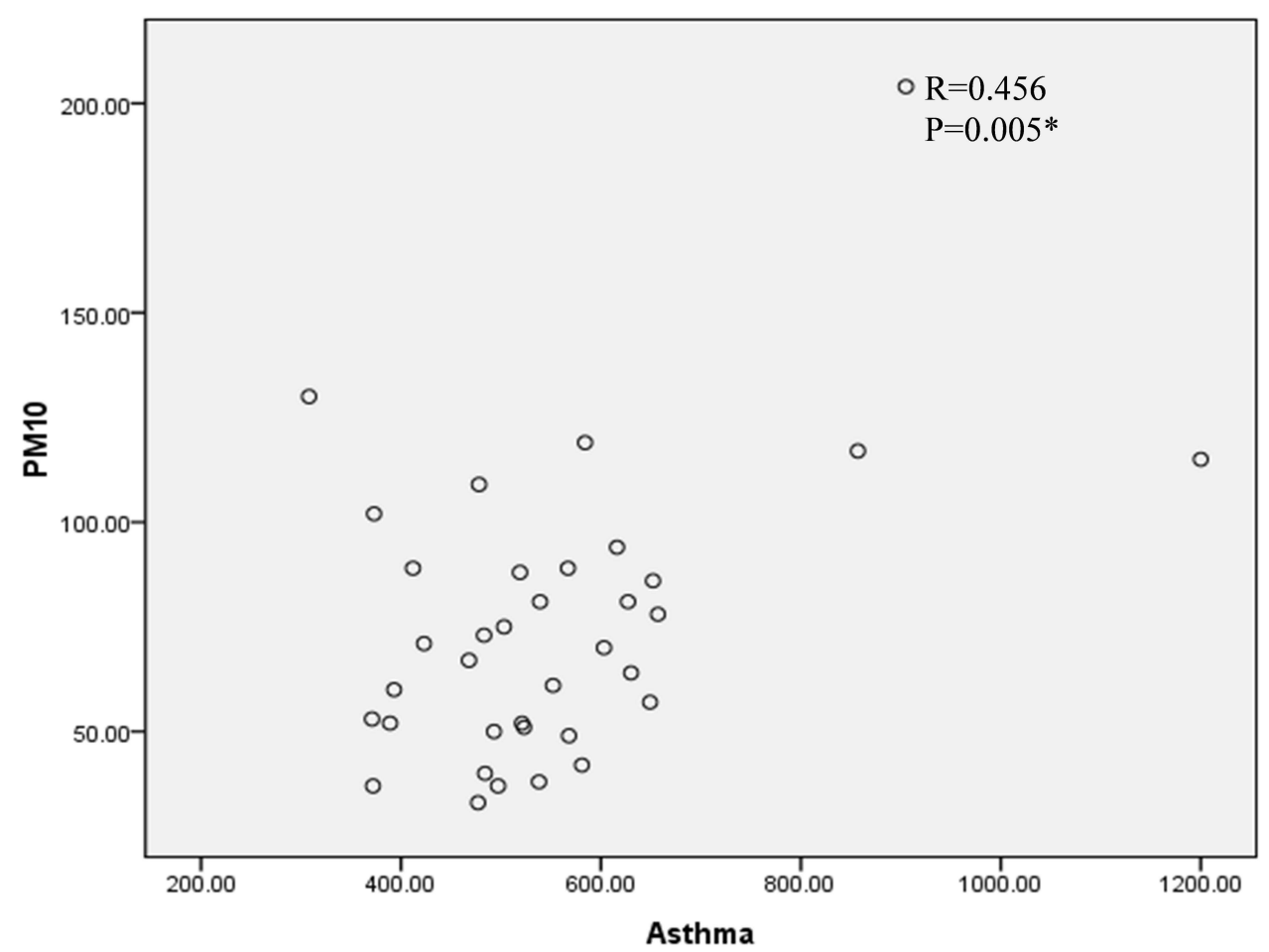

Figure 3 Correlation between the number of pediatric asthma patients and PMI0 from 2017 to $2019(* \mathrm{p}<0.05)$.

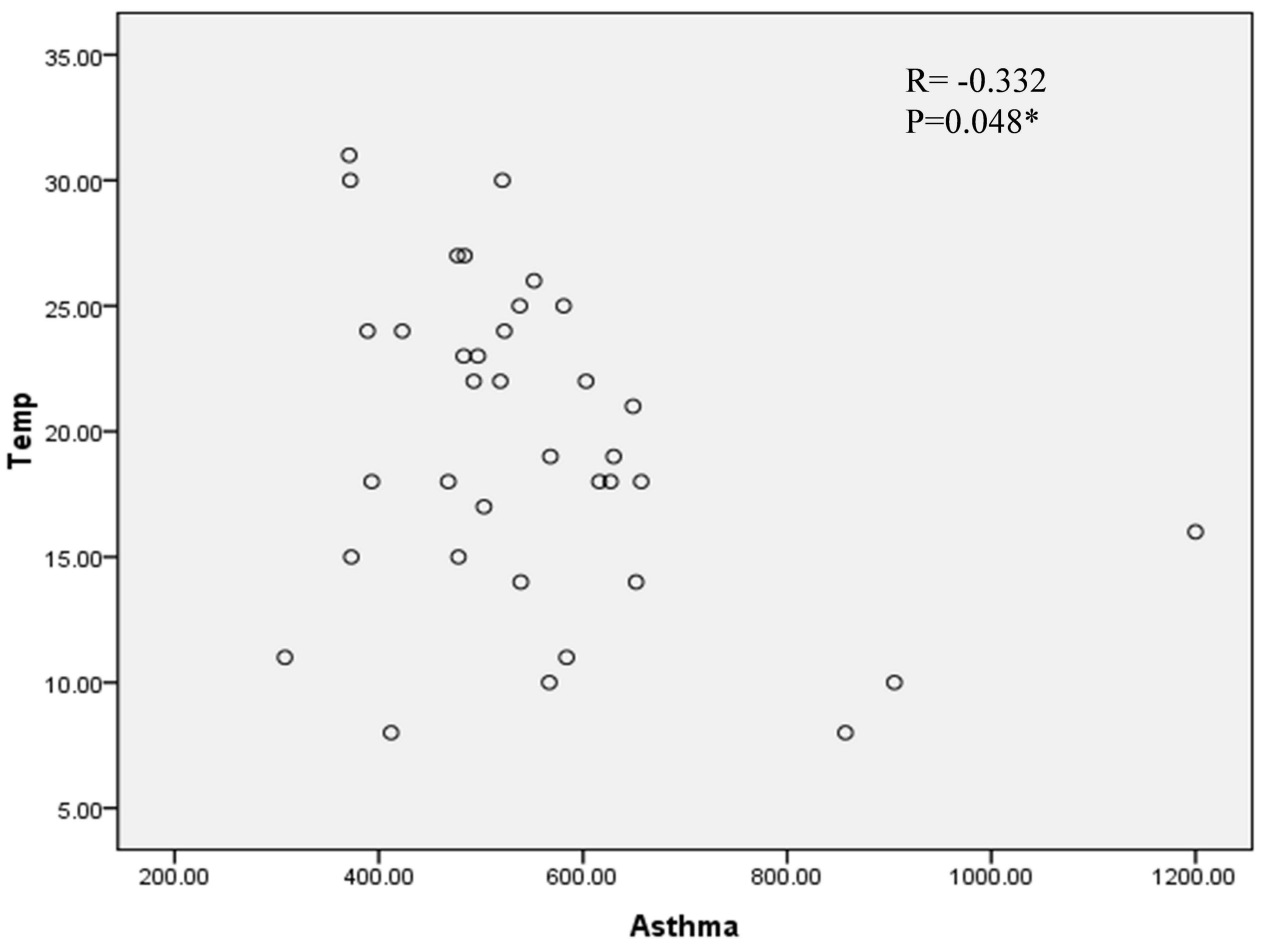

Figure 4 Correlation between the number of pediatric asthma patients and temperature from 2017 to $2019\left(*^{*}<0.05\right)$. 


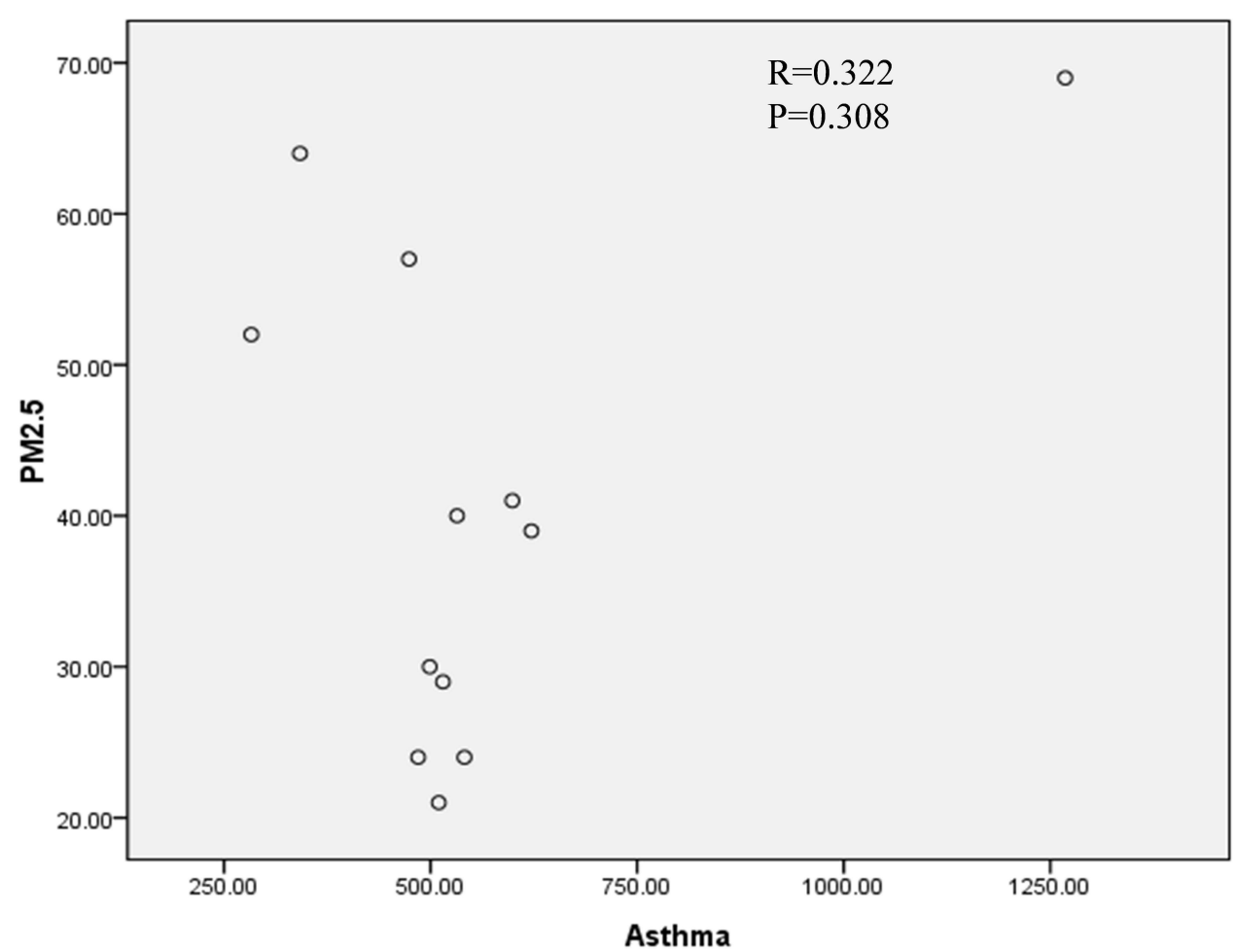

Figure 5 Correlation between the number of pediatric asthma patients and PM2.5 in 2020.

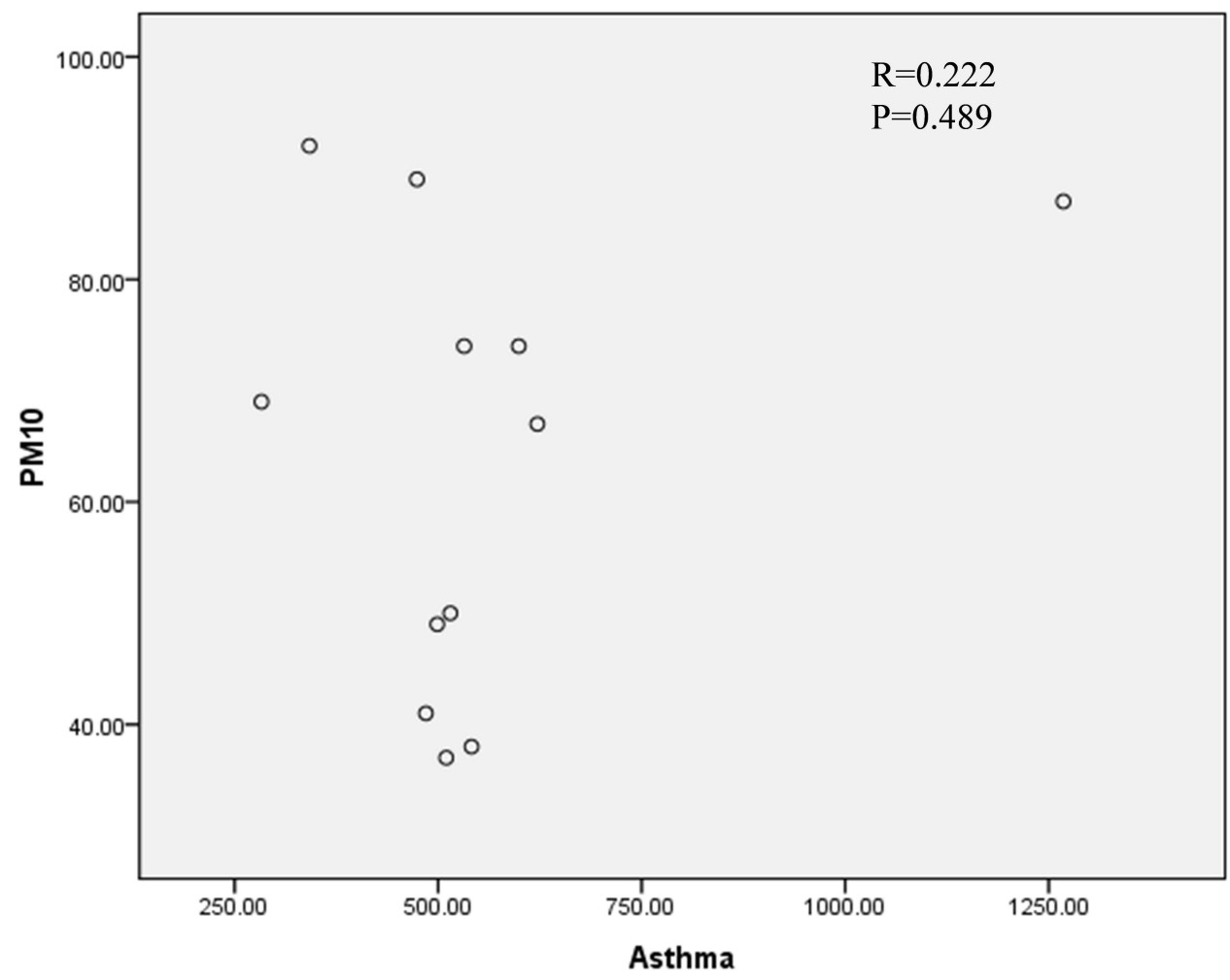

Figure 6 Correlation between the number of pediatric asthma patients and PMIO in 2020. 


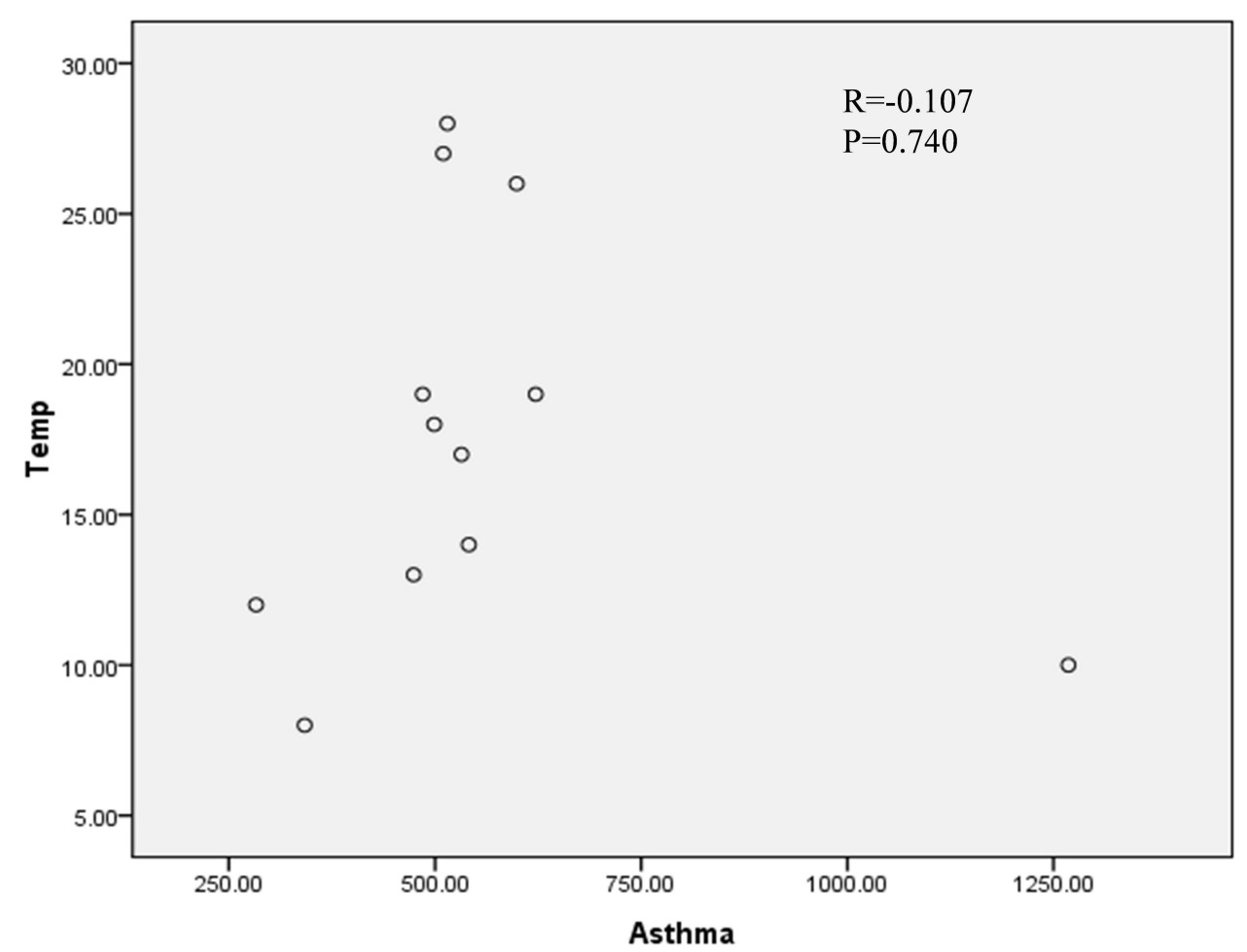

Figure 7 Correlation between the number of pediatric asthma patients and temperature in 2020 . (Asthma= the number of pediatric asthma patients, Temp= temperature).

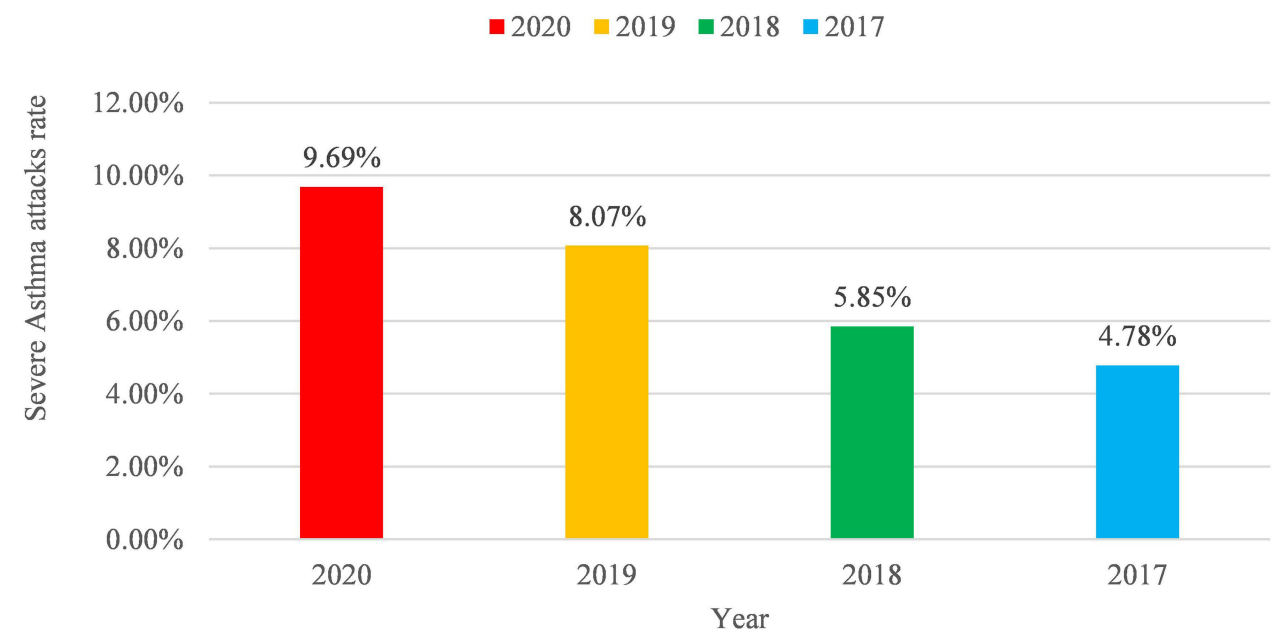

Figure 8 The rate of severe asthma attacks in 2017-2020.

$(F=86.3918, \mathrm{p}<0.001)$. (Figure 9). The incidence of $19.18 \%$ in September 2020 was markedly higher than in the previous year.

The number of patients failing to receive their scheduled treatment was no difference in 2018-2020 $(F=1.245$ $\mathrm{P}=0.496$ ) (Figure 10). Significantly more patients failed to attend pulmonary function tests in 2020 than in 2017-2019 $(F=418.3 P<0.001)$ (Figure 10).

\section{Discussion}

Bronchial asthma (asthma) is the most common chronic respiratory disease among children. In recent years, the incidence of pediatric asthma has exceeded that of adult asthma, and the number of pediatric asthma continues to increase around the world. In the 20 years up to 2010, the incidence of bronchial asthma in mainland China has grown by $147.9 \%$, affecting $3.02 \%$ of the 


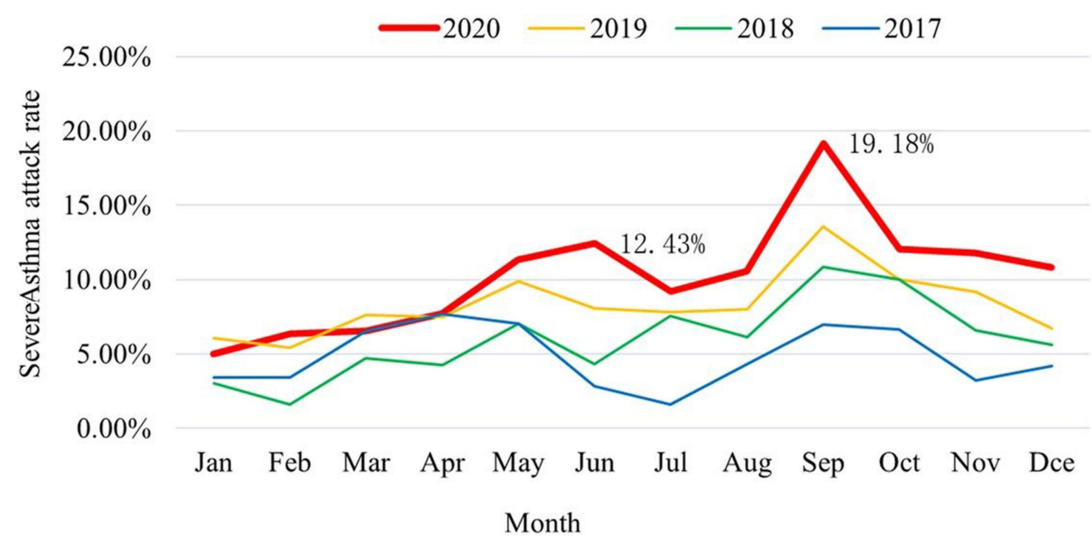

Figure 9 Monthly rate of severe asthma attacks in 2017-2020.

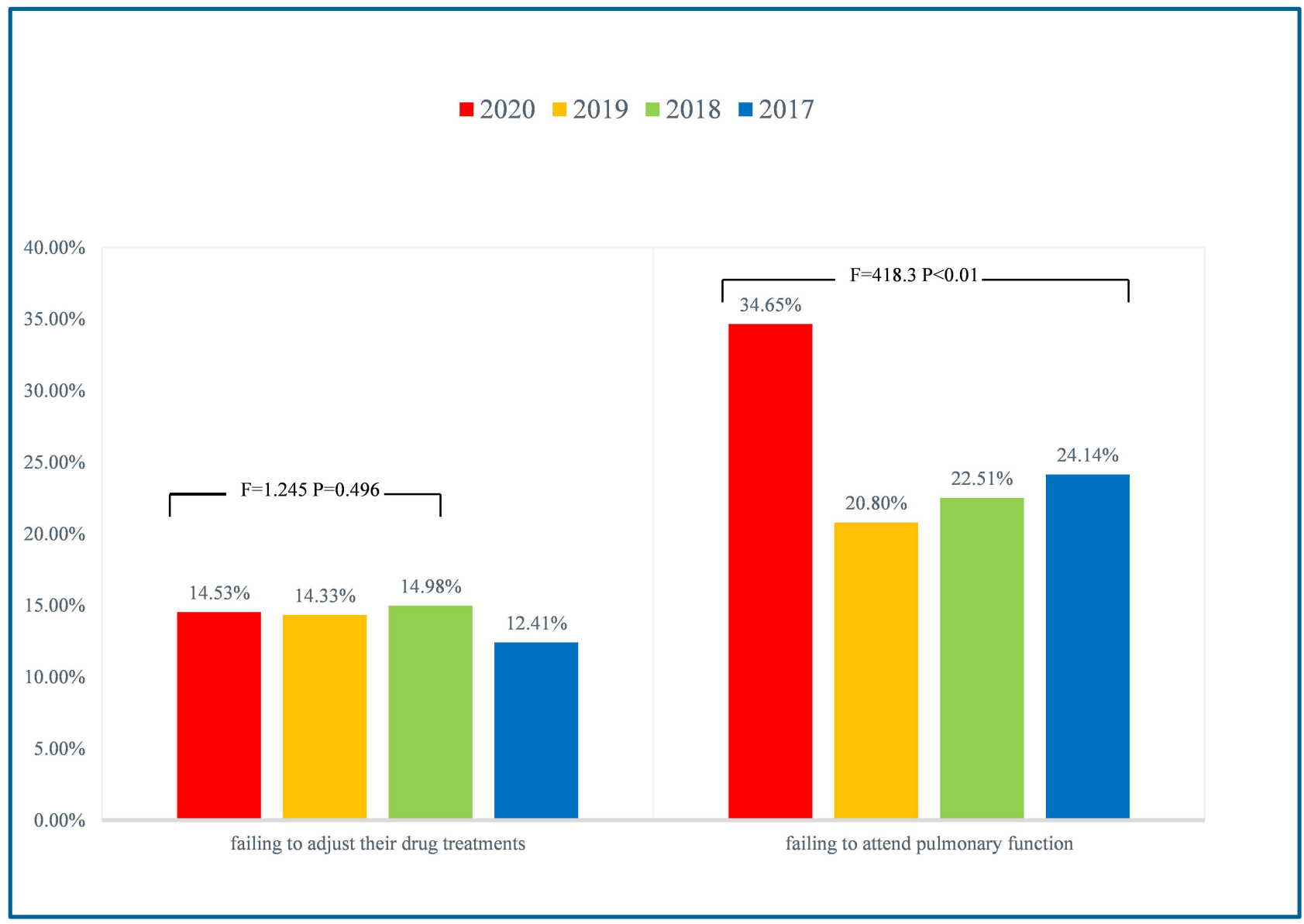

Figure 10 The percentage of patients failing to adjust their drug treatments and attend pulmonary function tests in 2017-2020.

population. ${ }^{3}$ Although asthma is currently incurable, good management of the disease can effectively control progression, minimizing the side effects and improving long-term quality of life. Working against this, however, is the COVID-19 pandemic, which has severely threatened public health by infecting more than
3 million people, and it has exacted great economic losses around the world. In this context, we investigated the asthma situations of children in Chengdu during the COVID-19 pandemic in order to provide a reference for future management and improvement of pediatric asthma. 
From the data on asthma patients aged 3-18 years in Chengdu, the overall number of patients remained steady in the period of 2017-2020. From 2017 to 2019, the number of asthma cases positively correlated with $\mathrm{PM}_{2.5}$ and $\mathrm{PM}_{10}$ levels, and it negatively correlated with temperature. These results are consistent with previous work. ${ }^{4}$ Chengdu is located in the southwest part of China, with a humid, foggy winter and a freezing, dry autumn. The dry conditions can make people less comfortable, compromising homeostasis in the human body and increasing risk of asthma. This may explain why the number of asthma cases usually peaks in January and February. Surprisingly, the number of asthma cases in 2020 was found to be unrelated to the air quality and temperature. It is suspected wearing a mask reduces the impact of air pollution and climate on asthma.

In order to mitigate COVID-19 disease transmission, unprecedented disease prevention measures were implemented in Chengdu, as in other places in China. The "Urgent Handling of Public Health Emergency Responses" was activated on 24 January 2020, and the disease prevention guidelines for primary, secondary and tertiary education were published in February 2020, which postponed the opening of schools. We suspect that the policies increased citizens' attention to the COVID-19 epidemic immediately. With limited outdoor activities and less exposure to cold air and air pollutants, crossinfection due to crowd gatherings has markedly decreased, resulting in a significant decline in the number of asthma cases in February 2020.

Recent research points out that during the COVID-19 pandemic, the number of emergency hospital visits due to severe asthma attack has significantly decreased. ${ }^{5}$ However, the analysis period of that study was relatively short. Here we retrospectively investigated cases of severe asthma attacks among children in 2020, with a focus on the long-term impact of COVID-19 pandemic on asthma patients. There will be more factors that influence the severe asthma attacks. In our study, we found that pediatric severe asthma attacks in 2020 were notably more frequent than in previous years (Figure 9). The differential pattern of the monthly pediatric incident rate in 2020 further supported the importance of using severe asthma attacks as a risk factor of the disease.

Owning to the disease prevention arrangements in 2020, schools in Chengdu have different start dates, and they only gradually resumed from May 2020. The normal start date of the summer semester is September. With more people gathering in schools and classrooms, the indoor air would be more static, which increases the chances of cross-infection of respiratory diseases and amplifies the risk of severe asthma attacks. Therefore, severe asthma attacks peaked in June and September 2020. In addition, people have adopted different more hygienic lifestyles such as wearing masks and frequently using disinfectants. Undoubtably, these practices can effectively curb viral transmission. However, there are studies showing that using N95 or KF94 masks increases the risk for chronic disease patients with impaired pulmonary function ${ }^{6}$ and that frequent disinfectant usage may affect the respiratory tract of newborns, increasing the risk of pediatric asthma. ${ }^{7}$ Zok et al showed that weekly usage of air fresheners may also be a risk factor of severe asthma onset. ${ }^{8}$ Considering the fact that $60-80 \%$ of allergic asthma cases involve pulmonary asthma, ${ }^{9}$ minimizing exposure to allergens should be the most effective method to control asthma. The frequent usage of alcoholic and chlorinated allergic disease prevention materials may be a risk factor of severe asthma attacks, contributing to the increase in the incidence of severe asthma attacks among children during the COVID-19 pandemic. Since certain disease prevention practices such as masks and disinfection are expected to persist even after the pandemic, we have to design ways to minimize the impacts of these measures on pediatric asthma patients so as to achieve effective asthma management.

At the same time, psychological and emotional factors are also important factors that may affect asthma management of the pediatric patients. ${ }^{10}$ During the COVID-19 pandemic, $7-53 \%$ of the individuals in China have experienced a psychological crisis. Although children may have experienced less fear towards COVID-19 due to their limited knowledge of the disease, their prolonged isolation, reduced exercise and the negative emotions of the family members may contribute to negative emotions among pediatric asthma patients, which may affect asthma control of pediatric asthma patients. ${ }^{11-13}$

Certainly, the parents maybe postpone hospital visits because they were scared of the risks of exposing their child or themselves to COVID-19, unless their child manifested severe asthma attacks. The fact is that the proportion of severe cases increased while the total number maybe decreased relatively.

With the recent advance of e-commerce within the region, a paradigm shift has occurred from the traditional practice of purchasing drugs in the hospital and pharmacy 
to a practice of purchasing them online in Chengdu. If any clinical discomfort occurs, patients' family members may choose to purchase asthma drugs by themselves instead of attending follow-up medical examinations. From 2018 to 2020 , there is no difference in the number of pediatric asthma patients who did not return to the hospital to receive planned medical treatments. But during the COVID-19 pandemic in $2020,34.56 \%$ of the patients failed to attend their scheduled pulmonary function tests, which is markedly higher than in previous years. During the pandemic, pediatric asthma patients were advised to avoid frequent hospital visits and minimize the usage of equipment contacting the respiratory tract, ${ }^{14}$ resulting in more patients failing to attend pulmonary function tests. Without regular pulmonary function testing and timely adjustment of asthma drug administration, only relying on clinical diagnosis of pediatric asthma patients may not effectively manage the disease and it may contribute to severe asthma attacks. This further supports the importance of both clinical examination and pulmonary function tests for managing asthma. In addition, the fact that children did not assess their asthma condition using peak flow meters illustrates that telemedicine has yet to replace traditional face-to-face medical examination. Family pulmonary function testing surveillance and long-range medical examinations are important for chronic asthma management in pediatric patients.

\section{Conclusion}

Although currently there is no evidence showing that asthma is a risk factor contributing to the COVID-19 infection or severity of the disease, ${ }^{15}$ studies have found that good asthma management can reduce the risk of severe asthma attacks during the pandemic. ${ }^{16}$ Based on the present study, we propose that more emphasis should be placed on disease prevention facilities and on the impacts of environmental and psychological factors on asthma management when designing treatment plans. We recommend proactively implementing telemedicine in asthma management in order to ensure effective pediatric asthma management during sudden pandemics.

The major limitation of this study is that even though we recruited data from a Level 3 Grade A general pediatric hospital with patients coming from urban and rural areas of Chengdu, only one hospital was chosen. Thus, the sample may contain selection bias, and the results may not be generalizable to all patient populations.

\section{Abbreviations}

COVID-19, coronavirus disease 2019; PM 2.5, particulate matter in the air $<2.5 \mu \mathrm{m}$; PM 10 , Particulate matter in the air $<10 \mu \mathrm{m}$; N95 mask, NIOSH N95 respirator dust mask; KF94 mask, KF94 respirator dust mask; MFDS, Ministry of Food and Drug Safety.

\section{Data Sharing Statement}

Authors wish to share their data. Please contact the Author Lei Zhang,534167313@qq.com for any requests regarding the study data.

\section{Ethics Approval and Consent to Participate}

The retrospective studies were approved by the ethics committee of the Chengdu Women's and Children's Hospital, Sichuan, China. Ethics Number B2021(5) The patient consent to review their medical records was not required by the ethics committee, because no mention of the patient's privacy in the article. We affirm the study complies with the Declaration of Helsinki and keeps all patient data confidential.

\section{Author Contributions}

All authors made a significant contribution to the work reported, whether that is in the conception, study design, execution, acquisition of data, analysis and interpretation, or in all these areas; took part in drafting, revising or critically reviewing the article; gave final approval of the version to be published; have agreed on the journal to which the article has been submitted; and agree to be accountable for all aspects of the work.

\section{Funding}

There is no funding to report.

\section{Disclosure}

The authors have no conflicts of interest to declare.

\section{References}

1. Asthma group of Chinese Throacic Society. Guidelines for bronchial asthma prevent and management (2020 edition) Asthma group of Chinese Thoracic Society. Chin J Tuberc Respir Dis. 2020;43 (12):1023-1044.

2. The Editorial Board, Chinese Journal of Pediatrics; the Subspecialty Group of Respiratory Diseases, the Society of Pediatrics, Chinese Medical Association; the Children's Respiratory Professional Committee, the Society of Pediatrics of Chinese Medical Doctor Association. Recommendations for diagnosis and management of bronchial asthma in children (2020). Chin J Pediatr. 2020;58 (9):708-717. 
3. Xiang L, Zhao J, Zheng Y, et al. Uncontrolled asthma and its risk factors in Chinese children: a cross-sectional observational study. J Asthma. 2016;53(7):699-706. doi:10.3109/02770903.2016.114 4199

4. Lavigne É, Talarico R, van Donkeaar A, et al. Fine particulate matter concentration and composition and the incidence of childhood asthma. Environ Int. 2021;5(152):106486. doi:10.1016/j. envint.2021.106486

5. Kenyon CC, Hill DA, Henrickson SE, et al. Initial effects of the COVID-19 pandemic on pediatric asthma emergency department utilization. J Allergy Clin Immunol Pract. 2020;8(8):2774-2776. doi:10.1016/j.jaip.2020.05.045

6. Kyung SY, Jeong SH. Particulate-matter related respiratory diseases. Tuberc Respir Dis. 2020;83(2):116-121. doi:10.4046/trd.2019.0025

7. Parks J, McCandless L, Dharma C, et al. Association of use of cleaning products with respiratory health in a Canadian birth cohort. CMAJ. 2020;192(7):E154-E161. doi:10.1503/cmaj.190819

8. Zock JP, Plana E, Jarvis D, et al. The use of household cleaning sprays and adult asthma: an international longitudinal study. Am J Respir Crit Care Med. 2007;176(8):735-741. doi:10.1164/ rccm.200612-17930C

9. The Respiratory Allergy Group of Chinese Society of Allergy, The Asthma Group of Chinese Thoracic Society, Chinese Medical Association. Chinese guidelines for the diagnosis and treatment of allergic asthma (2019, the first edition). Chin J Intern Med. 2019;58 (9):636-649.
10. Tong X, Ning W, Lyu Ju H, et al. Analysis of the disease control level and its influencing factors in children with asthma. Chin J Women Child Health Res. 2020;31(10):1295-1300.

11. Wang $C$, Pan $R$, Wan $X$, et al. Immediate psychological responses and associated factors during the initial stage of the 2019 coronavirus disease (CoViD-19) epidemic among the general population in China. Int J Env Res Public Heal. 2020;17:pii: E17.

12. Li S, Wang Y, Xue J, Zhao N, Zhu T. The Impact of CoViD-19 epidemic declaration on psychological consequences: a study on active weibousers. Int J Environ Res Public Health. 2020;17.

13. Qiu J, Shen B, Zhao M, et al. A nationwide survey of psychological distress among Chinese people in the CoVID-19 epidemic: implications and policy recommendations. Gen Psychiatr. 2020;33(2): e100213. doi:10.1136/gpsych-2020-100213

14. Chen ZM, Fu JF, Shu Q, et al. Diagnosis and treatment recommendations for pediatric respiratory infection caused by the 2019 novel coronavirus. World J Pediatr. 2020;16(3):240-246. doi:10.1007/ s12519-020-00345-5

15. Castro-Rodriguez JA, Forno E. Asthma and COVID-19 in children: a systematic review and call for data. medRxiv. 2020;2020.

16. Shaker MS, Oppenheimer J, Grayson M, et al. COVID-19: pandemic contingency planning for the allergy and immunology clinic. J Allergy Clin Immunol Pract. 2020;26.

\section{Publish your work in this journal}

The Journal of Asthma and Allergy is an international, peer-reviewed open-access journal publishing original research, reports, editorials and commentaries on the following topics: Asthma; Pulmonary physiology; Asthma related clinical health; Clinical immunology and the immunological basis of disease; Pharmacological interventions and new therapies. The manuscript management system is completely online and includes a very quick and fair peer-review system, which is all easy to use. Visit http://www.dovepress.com/testimonials.php to read real quotes from published authors. 